\title{
Compreensão de Idosos sobre os Benefícios da Atividade Física
} The Perception of the Elderly on the Benefits of Physical Activity

\author{
VICENTE FIDÉLIX FERREIRA GOMES JÚNIOR ${ }^{1}$ \\ ATHUS BASTOS BRANDÃO ${ }^{1}$ \\ FRANCISCO JAIRO MEDEIROS DE ALMEIDA ${ }^{1}$ \\ JANAIISA GOMES DIAS DE OLIVEIRA²
}

\section{RESUMO}

Introdução: O crescimento da população idosa ampliou as políticas públicas, projetos e programas objetivando atender a saúde global dessa classe de indivíduos, tendo a atividade física um meio preventivo dos efeitos físicos nocivos causados pelo envelhecimento. Objetivo: analisar como um grupo de idosos praticantes de exercícios físicos compreendem os efeitos proporcionados pela prática física. Material e Métodos: Trata-se de um estudo transversal de análise qualitativa em nível exploratório descritivo, onde foi aplicado uma entrevista semiestruturada a qual contemplou tópicos referentes a percepção dos idosos quanto a relevância da prática de atividade física regular na prevenção à saúde. Foi utilizada a análise de conteúdo de Bardin, propondo as seguintes categorias de análise: Situação de Saúde, Motivação para o Exercício e Percepção do exercício como tratamento. Resultados: Quanto a Situação de Saúde, os idosos identificam os benefícios da atividade física. Em relação à Motivação para o Exercício, os mesmos mostraramse motivados no que se refere aos efeitos positivos sobre a saúde e a sociabilização. Na percepção do exercício como tratamento, a maioria foi incentivada a praticar exercícios físicos por profissional de saúde ou indivíduos provenientes de seu contexto social. Conclusão: Pode-se constatar que os participantes da pesquisa, compreendem os benefícios de um programa de atividade física sobre sua saúde física e mental.

\section{DESCRITORES}

Saúde do Idoso; Exercício; Qualidade de Vida.

\begin{abstract}
Introduction: The increasing elderly population has contributed to broader public policies, projects and programs targeting their overall health. Physical activity is an important measure to prevent the harmful physical effects caused by aging. Objective: To analyze how a group of elderlies practitioners of exercise understand the effects provided by the practice of physical exercise. Material and Methods: This was a cross-sectional, qualitative, descriptive, exploratory study using a semi-structured interview concerning the elderly's perception on the importance of regular physical activity for health promotion. The Bardin's content analysis was used, based on which the following analysis categories were proposed: health condition; motivation to exercise; and perception of exercise as a treatment. Results: The elderly notice the benefits of physical activity on their health condition. Regarding motivation to exercise, they proved to be motivated in relation to the positive effects on health and socialization. As to the perception of exercise as a treatment, most respondents were encouraged by a health professional or individuals from their social context to practice physical exercise. Conclusion: The study participants understand the benefits of a physical activity program on their physical and mental health.
\end{abstract}

\section{DESCROPTORS}

Health of the Elderly; Exercise; Quality of Life.

1 Acadêmicos bolsistas do curso de fisioterapia do Instituto Superior de Teologia Aplicada - Faculdades INTA. Brasil. Sobral/CE, Brasil.
2 Professora orientadora curso de fisioterapia do Instituto Superior de Teologia Aplicada - Faculdades INTA. Brasil. Sobral/CE, Brasil. 
$\mathrm{O}$ potencial crescimento da população idosa fez surgir a necessidade de ampliação de políticas públicas, projetos e programas voltados a esta população com o objetivo de atender às necessidades física, mental e social desses indivíduos, sendo a atividade física um meio preventivo dos efeitos nocivos causados pelas implicações deletérias do envelhecimento ${ }^{1}$.

Estes três fatores marcam esse processo e podem se apresentar como maior prevalência de patologias crônicas, tendência ao isolamento e fragilidade às condições sociais, responsáveis por desencadear um aspecto negativo no idoso, culminando em solidão e caráter depressivo ${ }^{2}$.

A atividade física regular tem reflexos positivos sobre a melhoria da capacidade funcional e as habilidades físicas em indivíduos idosos, auxiliando a reduzir as taxas de dependência para realização de suas atividades de vida diária (AVDs), aspecto físico débil e declínio em sua esfera social, promovendo a interação e desenvolvimento de um estilo de vida mais saudável, deixando-o fisicamente ativo e melhorando sua qualidade de vida $^{3,4}$.

Dessa forma, a atividade física consegue promover a longevidade, melhora a cognição, o estado mental e os níveis de humor, o que resulta na redução dos níveis de depressão e isolamento 5 .

Contudo, a mudança no estilo de vida de idosos em relação à prática regular de atividades físicas, é uma importante forma de reduzir os efeitos prejudiciais sobre a sua funcionalidade, característicos da velhice e do sedentarismo ${ }^{6}$.

A Atenção Básica em Saúde, assim como os profissionais que a compõem, conta com programas que incentivam a mudança de maus hábitos de vida, como o de uma vida sedentária, na tentativa de reduzir e/ou controlar a prevalência de doenças crônicas relacionadas a terceira idade, tendo significante impacto em relação a saúde dessa população, diminuindo os custos gastos para manutenção da mesma ${ }^{6}$.

Entretanto, são vários os fatores que levam os idosos a aderirem um programa regular de exercícios físicos, e o que se destaca é a busca por uma vida saudável. Porém, sabe-se que para obter os benefícios pretendidos é necessária uma continuidade dessas atividades, onde o indivíduo deve ser constantemente estimulado intrinsecamente ou extrinsecamente, mantendo uma linearidade e consequente aumento do seu condicionamento físico? .

Além disso, a disposição de atividades em grupo permite que esses idosos interajam entre si, contribuindo no desenvolvimento de aspectos emocionais, comportamentais, entre outros, indo além dos momentos de lazer e esforço físico ${ }^{8 .}$
Desta forma, já que a prática de exercício físico previne e condiciona os idosos, contra as doenças desencadeadas pela inatividade, além de propiciar benefícios psicossociais na terceira idade, este estudo buscou analisar a compreensão de um grupo de idosos praticantes de exercícios físicos sobre os potenciais efeitos proporcionados pela atividade física.

\section{MATERIAL E MÉTODOS}

Trata-se de um estudo transversal utilizando uma abordagem qualitativa em nível exploratório descritivo, realizado com um grupo de idosos praticantes de exercícios físicos localizados no bairro Alto do Cristo, na cidade de Sobral-CE, onde se desenvolve o Programa de Atividades Físicas com Idosos Ativos.

Este programa incluiu atividades físicas aeróbicas, tais como caminhadas e ginástica aeróbica, com duração de $60 \mathrm{~min}$, durante duas vezes por semana, sendo o grupo supervisionado por educador físico.

A amostra foi não probabilística intencional, por conveniência, totalizando um grupo de 13 participantes idosos, com idade igual ou superior a 60 anos, portadores de atestado médico para aptidão física e assinatura do Termo de Consentimento Livre e Esclarecido. Excluiuse do estudo idosos não assíduos, com idade igual ou inferior a 59 anos e não assinatura do Termo de Consentimento Livre e Esclarecido.

Neste estudo aplicou-se uma entrevista semiestruturada que contemplava tópicos referentes a percepção dos idosos sobre a relevância da prática de atividade física regular na prevenção da saúde, tais como: aspectos da vida antes e após o grupo de atividade física, motivação, indicação da prática física entre outros.

Os idosos foram identificados por meio de um código alfanumérico individual, sendo utilizada a letra e para o significado de - entrevistado (a), em seguida, o número do entrevistado de acordo com a ordem em que foram organizadas as entrevistas, as consoantes $\mathrm{Me} \mathrm{F}$, indicando os gêneros masculino e feminino respectivamente. $\mathrm{O}$ exemplo a seguir demonstra como serão expostos a identificação nos depoimentos dos participantes: E1F - entrevistado 1 do sexo feminino.

Para a análise dos dados, foi utilizado a técnica de análise de conteúdo proposta por Bardin, que consiste no desvendamento dos centros de sentido compostos por uma comunicação, cuja presença e frequência tenham significado para objetivo analítico analisado. Esta é realizada em três etapas: a pré-análise, a exploração do material e o tratamento dos resultados obtidos e interpretação9 . 
Este estudo atende as recomendações da Resolução 466/2012 do Conselho Nacional de Saúde, referente ás pesquisas com seres humanos, o estudo foi avaliado e aprovado pelo comitê de ética e pesquisa da Universidade Estadual Vale do Acaraú - UVA com o parecer número 943.593 .

\section{RESULTADOS E DISCUSSÕES}

Para realização do presente estudo foram selecionados 13 indivíduos provenientes de um grupo de idosos da cidade de Sobral-CE, bairro Alto do Cristo, com idade média de 66 anos, com maior prevalência do público feminino, sendo 12 mulheres e 1 do sexo masculino. Os participantes apresentavam no mínimo uma doença de cunho cardiovascular, metabólico ou de origem osteomuscular.

As interpretações e análises foram organizadas em três categorias que continham recortes dos depoimentos dos participantes. As categorias foram: Situação de Saúde, Motivação para o Exercício e Percepção do exercício como tratamento.

\section{CATEGORIAS}

\section{SITUAÇÃO DE SAÚDE}

Esta categoria abordou a percepção dos idosos sobre sua vida antes de iniciarem a prática de atividade física regular, bem como identificar se a prática de exercícios influencia ou não a qualidade de vida do entrevistado, de modo geral.

A maioria dos entrevistados referiram possuir limitações físicas, as quais interferiam em sua autoconfiança, disposição, desempenho de suas funções físicas e que desencadearam manifestação de doenças cardiovasculares.

Além disso, identificou-se a presença de indicadores psicossociais tais como: depressão, isolamento e redução da autoestima. Estudos afirmam que estes fatores podem se relacionar com o universo multidimensional do envelhecimento, bem como o declínio de suas funções fisiológicas, fatores sociais ou perda de entes queridos ${ }^{10}$.

Identifica-se estas percepções nas seguintes falas:

"Eu era acomodada, sedentária. Sempre em casa e isso causava mais cansaço e me sentia solitária”. (E1F)

"Fiquei viúva, daí ficava mais sozinha em casa. Aí a atividade física me fez bem porque fez eu passar mais tempo com os amigos. “(E12F)

"Vivia hospitalizada, preguiçosa. Tinha dificuldade de levantar, caminhava com dificuldade, cansaço e dores nas pernas. “(E5F)

O sedentarismo é definido como um estado de baixo gasto energético, sendo este responsável pelo agravamento dos efeitos deletérios sofridos na terceira idade, influenciando sobre os padrões físico funcionais e psicossociais do indivíduo ${ }^{11}$.

Em um estudo com idosas que praticam atividade física ${ }^{12}$, frisou sobre a importância do envelhecimento ativo em populações idosas, sendo a capacidade funcional o mais importante preceito para possuir uma melhor qualidade de vida, dando uma maior independência ao indivíduo na execução de suas atividades de vida diária, garantindo um perfil mais saudável.

“(...) uma das principais formas de evitar, minimizar e/ou reverter a maioria dos declínios físicos, sociais e psicológicos que, frequentemente, acompanham o idoso, é a atividade física, demonstrando que ela está constantemente associada a melhoras significativas nas condições de saúde, como o controle do estresse, da obesidade, do diabetes, das doenças coronarianas e, principalmente, a melhora da aptidão funcional do idoso ${ }^{12}$. “

Em estudo que buscou identificar fatores preditivos ao suicídio na população idosa, concluiu-se que um ambiente coletivo, onde o longevo possa cultivar amizades se torna um local de proteção e de importante valor contra a depressão e o isolamento ${ }^{13}$. Nesse caso, o ambiente em grupo de exercícios, além de benefícios orgânicos e físicos, proporciona uma melhor socialização e distanciamento de agravos psicológicos.

Sobre como a atividade física influenciou na vida do idoso, observou-se um impacto positivo sobre a aptidão funcional, socialização, aumento da autoestima:

"Sinto que melhorou as dores, me sinto mais vigorosa. Consigo realizar melhor minhas atividades, mais disposta e me sinto bem comigo mesma. “(E5F)

"Aumentou minha disposição e diminuiu a quantidade de remédio para a pressão e para dor que eu tomava. "(E9M).

O entrevistado E9M, também relatou que além dos benefícios físicos e mentais, a atividade física regular auxiliou na redução do consumo de fármacos destinados 
ao controle de indicadores pressóricos e de doenças advindas do sedentarismo.

O uso de fármacos em idosos é maior do que na população mais jovem, tendo por sua finalidade sanar as alterações deletérias e comorbidades crônicas comuns nessa população ${ }^{14}$.

O uso de fármacos em idosos é maior do que na população jovem, e tem por finalidade sanar as alterações deletérias e comorbidades crônicas comuns nessa população ${ }^{14}$.

As intervenções não farmacológicas como o exercício físico, são empregadas na prevenção hipertensão arterial por conta de seu baixo custo, risco mínimo e eficácia no controle pressórico ${ }^{15}$. O exercício físico é mais que uma forma de prevenção, principalmente para patologias cardiovasculares e osteomusculares, ou seja, atuantes no processo de promoção da saúde, intensificando a integração da população idosa na busca por esse serviço ${ }^{16}$.

A efetividade do programa de exercícios, foi percebida através da redução ou inversão dos fatores agravantes à saúde do idoso, como a assiduidade em atividades extra grupais, relatos de melhora da autoestima e disposição do idoso para dar continuidade às atividades. Além disso, a percepção sobre a melhora do estado de saúde, também fora percebida através da redução na administração de fármacos, segundo os pesquisados.

\section{MOTIVAÇÃO PARA O EXERCÍCIO}

Esta categoria disserta sobre o que motiva o idoso a praticar atividades físicas regulares, conhecer os indicadores que o influenciaram a permanecer no grupo, bem como verificar a percepção física após a execução do programa.

As motivações mais frequentes se relacionaram com a presença de problemas de saúde de origem física ou emocional, como exposto nos depoimentos a seguir:

"Me foi indicado caminhada para reduzir as dores no joelho, controlar a pressão e o colesterol. "(E1F).

"Ficava muito tempo dentro de casa sem fazer nada, só assistindo televisão, me sentia mal, daí fui atrás de fazer exercício para prevenir as doenças. "(E4F).

"Tive problema com depressão então o médico mandou quando estava melhorando. "(E6F).

A atividade física é tida como uma forma de prevenção a doenças de cunho cardiovascular em idosos, mostrando-se benéfica na manutenção da saúde física e níveis de força muscular, assim como tem relação positiva na saúde psicológica, reduzindo o estresse e aumentando a autoestima, tendo boa influência sobre o aspecto cognitivo do idoso ${ }^{10}$.

Estudos destacam que a socialização, sensação de prazer e redução do estresse, como categorias de motivação alta, sendo o fator estético e competição itens que representam menor índice de motivação entre idosos praticantes de exercícios físicos ${ }^{17}$.

“(...) o aparecimento da dor e a diminuição das atividades de vida diária, em consequência das doenças, interferem sobre a autoestima, uma vez que a pessoa se sente menos auto eficaz e percebe diminuído seu poder de controlar aspectos da vida. "17

Em relação a percepção da realização da atividade todos os pesquisados relataram sensação de bem-estar físico e mental e sem sintomas patológicos.

Verificou-se um sentimento de apreço quanto as atividades em grupo, pois o meio coletivo, segundo os mesmos, atuava como incentivador para manter a adesão, melhorando assim sua disposição para as atividades cotidianas e autoestima, este fato pode ser constatado nas seguintes falas:

"No momento me sinto mais alegre e disposta no meio das amigas, me esqueço dos problemas de casa. " (E12F)

"Me sinto disposta para fazer outras atividades. Me sinto disposta para fazer as coisas de casa. " (E6F)

Em análise do recorte da fala do E10F, constatado que, mesmo indo com menor frequência às atividades físicas do grupo, melhorou suas dores corporais, como segue:

"Melhor. As dores que eu sinto parecem melhorar com o exercício, parece que some, mesmo fazendo pouco. “(E10F)

Estudos ressaltam que a atividade física regular, quando executada por pelo menos trinta minutos, na maior parte da semana, mostram evidências quanto aos benefícios físicos e metabólicos associados a pessoas com idade maior ou igual a 45 anos, influenciando diretamente em seu aspecto funcional e qualidade de vida ${ }^{18}$.

Os benefícios físicos e cardiovasculares do exercício se mostram com maior eficiência em atividades intervaladas, com durabilidade dos efeitos fisiológicos entre as 20 primeiras horas pós-exercício, em uma sessão, o que ressalta a importância da prática regular de atividades ${ }^{19}$. 
Logo, a prática de atividade física regular em grupo, proporciona o afastamento de injúrias osteomusculares e cardiovasculares provenientes da inatividade, bem como, o isolamento social, atuando positivamente na qualidade de vida dessa população.

\section{PERCEPÇÃO DO EXERCÍCIO COMO TRATAMENTO}

Essa categoria analisou a percepção dos idosos sobre quais os valores atribuídos à prática de exercícios em suas vidas, almejando a promoção de saúde. Esta, também focou informações quanto a indicação do programa, poder de decisão quanto a permanência no grupo.

Todos afirmaram se sentirem mais se exercitados fisicamente, evitando assim, doenças crônicas, além de melhoria do bom humor:

É a de me sentir melhor, ter mais amizades, além de me divertir bastante. Me ajuda muito na disposição, além de ocupar mais o meu tempo, pois ficava muito sozinha e agora já não fico mais. É bom “pra” animar a vida! “ (E1F)

Ajuda no combate à diabetes, colesterol e o cansaço. "(E4F)

Importante, pois me dá coragem, me deixa bemhumorada, permite a convivência com outras pessoas. " (E7F)

Em relação a indicação da realização de atividade física regular a outros idosos, maioria dos participantes inseridos no programa relataram afirmaram positivamente:

"Sim, para melhorar, como eu melhorei, porque é muito bom. " (E5F)

"Indico. Gostaria de ver e saber que outras pessoas se beneficiam de exercício físico. É como indicar um remédio. " (E6F)

Contudo, sobre quem indicou a prática houve divergência entre as respostas, alguns procuraram o grupo por si só, enquanto outros foram informados por meios de comunicação, como no caso da idosa E8F. Esta participante relatou ter ouvido uma informação sobre exercícios e saúde na televisão. Observa-se abaixo relatos relevantes:

"Ninguém. Sou uma das primeiras que começaram. Comecei porque vi o grupo se formando, daí fiquei interessada." (E1F)

"Interesse próprio. A iniciativa foi minha no início, mas ouvi antes falando na TV sobre os benefícios da atividade física. “( $\mathrm{E} 8 \mathrm{~F})$
Alguns dos idosos foram incentivados a praticar atividade física por encorajamento de amigos ou por profissionais da saúde (médico e agente comunitária em saúde):

"Os médicos. Porque sinto dores no peito, palpitação, melhorou um pouco com a atividade física. "(E7F)

Os seguintes participantes: E2F, E3F, E9M e E7F relataram que seus parentes e/ou amigos os influenciaram na tomada de decisão de procuraram grupos especializados de exercícios físicos direcionados para idosos.

O entrevistado E9M, referiu ter sido estimulado por profissionais da equipe mínima de saúde, ou seja, médico ou agente comunitário, em função de alguma patologia pré-estabelecida. Já os participantes: E11F e $\mathrm{E} 12 \mathrm{~F}$, foram encorajados por amigos que já participavam do grupo e por profissionais da saúde.

A contribuição de diferentes tipos de atividade física na mediação da relação de fatores ambientais sociais e físicos com qualidade de saúde mental em adultos de meia-idade e mais velhos, identificou-se que: os fatores determinantes para a prática contínua de atividade física em idosos relaciona-se ao completo bem-estar físico e psicológico dos indivíduos. Porém, o início e a manutenção dessa prática estão diretamente ligados a um adequado apoio social e encorajamento pela família e amigos para essa atitude ${ }^{20,21}$.

Em relação ao encaminhamento por profissionais da saúde ${ }^{22}$, deve-se sempre priorizar a prevenção de doenças, em idosos e o afastamento de possíveis causas que possam levar ao surgimento dessas. Assim, evitase a morbimortalidade, e proporciona um envelhecimento saudável com qualidade de vida.

O trabalho interdisciplinar entre diversos profissionais, permite o compartilhamento de casos clínicos e a elaboração de planos que atendem as carências de dada população. Essa característica se dá, inicialmente, nos níveis de atenção primária a saúde, onde se constitui a porta de entrada para os serviços públicos de saúde à população ${ }^{23}$.

Constatou-se por meio dos recortes supracitados que todos os entrevistados consideram a atividade física um meio de tratamento de manutenção da saúde, tendo iniciado sua prática acreditando alcançar os efeitos fisiológicos afirmados pelos médicos, e vistos gradativamente em seus organismos.

Ressalta-se a importância dos meios de comunicação como forma de difundir informações sobre saúde à população de forma compreensível e de fácil assimilação, estando disponíveis de formas diversificadas e bem elaboradas. Acredita-se que a 
estimulação por um profissional de saúde, dá maior credibilidade no que concerne a adesão da prática por motivos de saúde. A atuação do agente comunitário em saúde permite uma melhor configuração nesse quesito, pois o mesmo constrói um elo entre o nível básico de atenção à saúde e população.

Também houveram indivíduos que referiram participar do programa por iniciativa própria, levando em consideração o âmbito social, que, como já descrito anteriormente, é um fator decisivo para a sua inserção.

\section{CONCLUSÃO}

Este estudo analisou a compreensão de um grupo de idosos praticantes de exercícios físicos sobre os potenciais efeitos proporcionados por um programa de atividade física regular, constatando-se que os

\section{REFERÊNCIAS}

1. Souza DL, Vendrusculo R. Fatores determinantes para a continuidade da participação de idosos em programas de atividade física: a experiência dos participantes do projeto "Sem Fronteiras". Rev Bras Educ Fís Esporte. 2010; 24(1):95105.

2. Lourenço TM, Lenardt MH, Kletemberg DF, Seima MD, Tallmann AEC, Neu DKM. Capacidade funcional no idoso longevo: uma revisão integrativa. Rev Gaúcha Enferm. 2012; 33(2):176-85.

3. Nascimento CMC Ayan C, Cancela JM, Pereira JR, Andrade LP, Garuffi M, et al. Exercícios físicos generalizados, capacidade funcional e sintomas depressivos em idosos brasileiros. Rev Bras Cineanotropom Desempenho Hum. 2013; 15(4):486-97.

4. Deslandes, A. The biological clock keeps ticking, but exercise may turn it back. Arq Neuro-Psiquiatr. 2013; 71(2):113-18.

5. McNaughton SA, Crawford D, Ball K, Salmon J. Understanding determinants of nutrition, physical activity and quality of life among older adults: the Wellbeing, Eating and Exercise for a Long Life (WELL) study. Health Qual Life Outcomes. 2012; 10: 109-16.

6. Michelin E, Corrente JE, Burini RC. Fatores associados aos componentes de aptidão e nível de atividade física de usuários da Estratégia de Saúde da Família, Município de Botucatu, Estado de São Paulo, Brasil, 2006 a 2007. Epidemiol Serv Saúde. 2011; 20(4):471-80.

7. Maciel MG. Atividade física e funcionalidade do idoso. Motriz: Rev Educ Fis. 2010; 16(4):1024-32.

8. Tahan J, Carvalho ACD. Reflexões de Idosos Participantes de Grupos de Promoção de Saúde Acerca do Envelhecimento e da Qualidade de Vida. Rev Saúde Soc. 2010; 19(4):878-88.

9. Vergara SC. Réplica 2 - análise de conteúdo como técnica de análise de dados qualitativos no campo da administração: potencial e desafios. Rev Adm Contemp. 2011; 15(4):761-65.

10. Meurer ST, Benedetti TRB, Mazo GZ. Teoria da autodeterminação: compreensão dos fatores motivacionais e da autoestima de idosos praticantes de exercício físico. Rev Bras Ativ Fís. $2011 ; 16(1): 18-24$

11. Helseltine R, Skelton DA, Kendrick D, Morris RW, Griffin M, Haworth D. "Keeping Moving": factors associated with sedentary behaviour among older people recruited to an exercise promotion trial in general practice. BMC Family Practice. 2015; 16:67-76.

12. Cipriani NCS, Meurer ST, Benedetti TRB, Lopes MA. Aptidão funcional de idosas praticantes de atividade física. Rev Bras Cineantropom Desempenho Hum. 2010; 12(2):106-11.

13. Minayo MCS; CAVALCANTE FG. Suicídio entre pessoas idosas: revisão da literatura. Rev Saúde Públ. 2010; 44(4):750-7.

14. Farias RG; Santos SMA. Influência dos Determinantes do Envelhecimento Ativo entre Idosos mais idosos. Texto Contexto Enferm. 2012; 21(1):167-76. participantes relataram melhora física e psicológica após se exercitarem, desta forma, sugere-se que o exercício físico é indispensável para o controle da saúde.

Ademais, os pesquisados, em sua maioria, foram incentivados a praticar exercícios físicos tanto por profissional de saúde, por amigos e outros participantes. Houveram casos em que alguns, por si só, buscaram se exercitar, tendo como motivação maior, a socialização com o grupo e o sentimento de bem-estar.

Portanto, tem-se o envelhecimento ativo, como um fator primordial para o prolongamento da vida com qualidade para idosos, uma vez que o mesmo proporciona o afastamento da dependência funcional e de desarranjos relacionadas ao estado psicossocial do indivíduo, como depressão e isolamento, que conduzem a uma vida sedentária, predispondo ao surgimento de doenças cardiovasculares e de outras disfunções sistêmicas.

15. Rêgo ARON, Gomes ALM, Veras RP, Andrade Júnior ED, Nunes RAM, Dantas EHM. Pressão Arterial após Programa de Exercício Físico Supervisionado em Mulheres Idosas Hipertensas. Rev Bras Med Esporte. 2011; 17(5):300-04.

16. Virtuoso JF, Mazo GZ, Menezes EC, Cardoso AS, Dias RG, Balbê GP. Perfil de morbidade referida e padrão de acesso a serviços de saúde por idosos praticantes de atividade física. Ciên Saúde Colet. 2012; 17(1):23-31.

17. Molanorouzi K, Khoo S, Morris T. Motives for adult participation in physical activity: type of activity, age, and gender. BMC Public Health. 2015; 15(66):1-12. Doi: 10.1186/s12889-015-14297.

18. Plotnikoff RC, Costigan SA, Short C, Grunseit A, James E, Johnson N, et al. Factors Associated with Higher Sitting Time in General, Chronic Disease, and Psychologically-Distressed, Adult Populations: Findings from the 45 \& Up Study. PLOS ONE. 2015; 10(6): e0127689. Doi: 10.1371/ ONE. 2015; $10(6)$ :

19. Carvalho RST, Pires CMR, Junqueira GC, Freitas D, MarchiAlves LM. Magnitude e Duração da Resposta Hipotensora em Hipertensos: Exercício Contínuo e Intervalado. Arq Bras Cardiol. 2014; 104(3):234-41. Doi: 10.5935/abc.20140193.

20. Van Dyck DV, Teychenne M, McNaughton SA, De Bourdeaudhuij I, Salmon J. Relationship of the perceived social and physical environment with mental health-related quality of life in middleaged and older adults: mediating effects of physical activity. PLoS One. 2015; 10(3):e0120475.

21. Pereira RJ, Cotta RMM, Franceschini SCC, Ribeiro RCL, Tinoco ALA, Rosado LEFPL, et al. Análise do Perfil Sociosanitário de Idosos: a importância do Programa Saúde da Família. Rev Med Minas Gerais. 2010; 20(1):5-15.

22. Souza MC, Bomfim AS, Souza JN, Franco TB. Fisioterapia e Núcleo de Apoio a Saúde da Familia: conhecimento, ferramentas e desafios. Mundo Saúde. 2013; 37(2):178-84.

23. Costa ACO, Moimaz SAS, Garbin AJI, Garbin CAS. Plano de carreira, cargos e salários: ferramenta favorável à valorização dos recursos humanos em saúde pública. Odontol Clínient. 2010; 9(2):119-23.

\section{Correspondência}

Vicente Fidelix Ferreira Gomes Júnior

Rua Antônio Crisóstomo de Melo, 327.

Sobral - Ceará - Brasil - CEP: 62010-550

E-mail: vincevince13@gmail.com 